\title{
High Pressure Band Structure, Density of States, Structural Phase Transition and Metallization in CdS
}

\author{
J. Jesse Pius ${ }^{1}$, A. Lekshmi ${ }^{2}$, C. Nirmala Louis ${ }^{2, *}$ \\ ${ }^{1}$ Rohini College of Engineering, Nagercoil, Kanyakumari District, India \\ ${ }^{2}$ Research Center in Physics, Holy Cross College, India
}

Copyright $\mathrm{O} 2017$ by authors, all rights reserved. Authors agree that this article remains permanently open access under the terms of the Creative Commons Attribution License 4.0 International License

\begin{abstract}
The electronic band structure, density of states, metallization and structural phase transition of cubic zinc blende type cadmium sulphide $(\mathrm{CdS})$ is investigated using the full potential linear muffin-tin orbital (FP-LMTO) method. The ground state properties and band gap values are compared with the experimental results. The equilibrium lattice constant, bulk modulus and its pressure derivative and the phase transition pressure at which the compounds undergo structural phase transition from $\mathrm{ZnS}$ to $\mathrm{NaCl}$ are predicted from the total energy calculations. The density of states at the Fermi level $\left(\mathrm{N}\left(\mathrm{E}_{\mathrm{F}}\right)\right)$ gets enhanced under pressure, which leads to metallization in CdS. The pressure corresponding to structural phase transition from $\mathrm{ZnS}$ structure (B3) to the $\mathrm{NaCl}$ structure (B1) is 0.0168 Mbar in CdS. The metallization pressure PM is 6.9133 Mbar.
\end{abstract}

Keywords Band Structure, Metallization, Structural Transition, CdS, High Pressure

\section{Introduction}

The theoretical study on electronic band structure, density of states, phase stability and structural changes of crystalline materials play a basic role in Theoretical and Computational Physics as well as Chemical and Material Engineering, because the better understanding of the various physical and chemical properties of solids mainly depend upon their structure. Currently there is lot of interest in the study of structural changes and phase stability of materials under pressure. The physical and chemical properties of materials undergo a variety of changes when they are subjected to pressure [1].

In recent years there has been an increased interest in the effect of high pressure on materials [2]. Geologists have probed the behavior of materials under pressure for learning the physico-chemistry of materials near the center of the earth. There is a continued advancement and interest in the high pressure studies due to the development of different designs of diamond anvil cell (DAC). With a modern DAC, it is possible to reach pressures of $2 \mathrm{Mbar}(200 \mathrm{GPa})$ routinely and pressures of $5 \mathrm{Mbar}(500 \mathrm{GPa})$ or higher is achievable [3]. At such pressures, materials are reduced to fractions of their original volumes. With this reduction in inter atomic distances; significant changes in bonding and structure as well as other properties take place. The increase of pressure means the significant decrease in volume, which results in the change of electronic states and crystal structure. It is an interesting phenomenon that pressure can change the electrical properties of matter. Generally it narrows the energy bandgaps of matter and hence the matter could transform from semiconductor to metal [1-8].

Recently, pressure induced phase transition of $\mathrm{CdS}$ was investigated using Raman spectroscopy and in situ electrical resistivity measurement on DAC by Wang et al [4]. In this study pressure induced phase transition of $\mathrm{CdS}$ was investigated by Raman spectroscopy and electrical resistivity measurement on DAC. Both of the experiments suggested the occurrence of the CdS phase transition from WZ to intermediate to RS phase. Besides, electrical resistivity measurements indicated that $\mathrm{CdS}$ undergoes semiconductor - metal phase transition under high pressure [4]. As a group II-VI semiconductor, cadmium sulfide has gained wide recognition because of its outstanding optical-electronic properties and polymorphic structural transformations. It has a direct band gap of $2.4 \mathrm{eV}$ that lends itself to many applications in light detectors, forming quantum dots, and passivating the surfaces of other materials. Owing to the thermal stability and the colour in yellow, it can form pigments in colors ranging from deep red to yellow with the addition of CdTe [5].

The pressure-induced phase transitions of cadmium sulfide semiconductor in both zinc-blende and wurtzite structures were investigated by ab initio plane-wave pseudo potential density functional theory with the local density approximation by Tan etal [5]. In this study, the effects of the pressure on the elastic constants and mechanical properties were calculated. The high-pressure phase transitions in II-VI 
semiconductors were given by Vladimir V. Shchennikov etal [6]. The indirect energy gaps of $\mathrm{NaCl}$-structured $\mathrm{CdS}$ and CdSe are measured using absorption spectroscopy to approximately 55 and $42 \mathrm{GPa}$ by Cervantes etal [7]. There is no high pressure studies related to metallization in CdS. This motivated us to take up the present investigation on CdS.

The electronic configuration of $\mathrm{Cd}$ and $\mathrm{S}$ are $[\mathrm{Kr}] 4 \mathrm{~d}^{10} 5 \mathrm{~s}^{2}$ $(\mathrm{Z}=48)$ and $[\mathrm{Ne}] 3 \mathrm{~s}^{2} 3 \mathrm{p}^{4}(\mathrm{Z}=16)$ respectively. The valence electronic configurations $4 \mathrm{~d}^{10} 5 \mathrm{~s}^{2}$ for $\mathrm{Cd}$ and $3 \mathrm{~s}^{2} 3 \mathrm{p}^{4}$ for $\mathrm{S}$ are chosen in our calculation. We have obtained the band structures and density of states of CdS corresponding to various pressures using the full potential linear muffin-tin orbital (FP-LMTO) method [1,8]. The calculated total energies were fitted to the Murnaghan's equation of state(EOS) [1], todetermine the phase transition pressure and other ground state properties. Generally sulphide compounds $(\mathrm{ZnS}$ and $\mathrm{CdS})$ crystallize in zinc blende structure $(\mathrm{ZnS})[9$, 10]. Group II-VI compounds undergo structural phase transition from zinc blende to $\mathrm{NaCl}$ structure prior to metallization. The phenomena of metallization at high pressure $(\mathrm{NaCl})$ structure of $\mathrm{CdS}$ are analyzed. We give the details of the calculational procedure and electronic band structure corresponding to various pressures in Section 2. In Section 3, the ground-state properties, structural phase transition and metallization are discussed. Concluding remarks are given in Section 4.

\section{Band Structure and Density of States}

\subsection{Calculational Procedure}

Group II-VI semiconductor CdS crystallizes in zinc blende structure under ambient conditions and undergo structural phase transition from $\mathrm{ZnS}$ to $\mathrm{NaCl}$ structure. The electronic band structure calculations were performed for $\mathrm{CdS}$ corresponding to different reduced volumes both in $\mathrm{ZnS}$ and $\mathrm{NaCl}$ structures, by the first-principle linear muffin-tin orbital (FP-LMTO) method. We have used FP-LMTO method with in generalized gradient approximation (GGA) [1]. We have used the exchange-correlation potential of Ceperley and Alder as parametrized by Perdew and Zunger [1].We give here only the calculational details. The electronic configuration of $\mathrm{Cd}$ and $\mathrm{S}$ are $[\mathrm{Kr}] 4 \mathrm{~d}^{10} 5 \mathrm{~s}^{2}(\mathrm{Z}=48)$ and $[\mathrm{Ne}] 3 \mathrm{~s}^{2} 3 \mathrm{p}^{4}(\mathrm{Z}=16)$ respectively. The valence electronic configurations chosen in our calculation are $4 \mathrm{~d}^{10} 5 \mathrm{~s}^{2}$ for $\mathrm{Cd}$ and $3 s^{2} 3 p^{4}$ for $S$.There are 18 valence electrons contributing to the valence bands [1]. The final energy convergence is within $10^{-5}$ Ry. The calculated total energies were fitted to Murnaghan's equation of state (EOS) [2], to determine the bulk modulus and pressure derivative of bulk modulus Bo'. Theoretical studies of cohesive, structural and vibrational properties of solids under pressure are performed by means of FP-LMTO calculations [8-10]. We have obtained, (i) normal pressure band structure and density of states of $C d S$ (with $\mathrm{ZnS}$ structure),

(ii) high pressure band structure and density of states of $C d S$ (with $\mathrm{NaCl}$ structure),

Also, we have analyzed the structural phase transition from $\mathrm{ZnS} \rightarrow \mathrm{NaCl}$ structure and metallization. The radii of the Wigner-Seitz sphere are chosen according to the criterion for charge transfer from cation to the anion [11]. The entire Brillouin zone is partitioned into 4096 cubical volume elements and $E(k)$ are computed for the $k$-points located at the center of each volume element. But because of the symmetry considerations this involves only $145 k$ points for both $\mathrm{ZnS}$ and $\mathrm{NaCl}$ structures, which are uniformly distributed in the 1/48-th of the first Brillouin zone [12]. In the calculation of total energy with $\mathrm{NaCl}$ structure, two empty spheres (ES) are positioned along the body diagonal

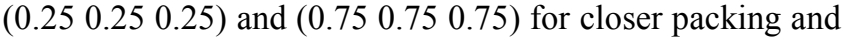
with the $\mathrm{ZnS}$ structure two empty spheres are introduced at positions $(0.50 .50 .5)$ and $(0.750 .750 .75)$ in such a way that they do not break the crystal symmetry. The calculated total energies are fitted to Murnaghan's equation of state (EOS) [13], to determine the pressure derivative of bulk modulus $\mathrm{B}_{\mathrm{o}}{ }^{1}$, phase-transition pressure and other ground-state properties $[14,15]$. The bulk modulus is a property of the material which defines its resistance to volume change when compressed. The cause for phase transition and the relation between phase transition pressure and atomic radii are deduced from band structure investigation $[16,17]$.

\subsection{Band Structure and Density of States of CdS under Pressure}

The band structures and density of states for $C d S$ is computed for various reduced volumes ranging from $V / V_{o}=1.0$ to 0.3 in steps of 0.05 (Figs.1 to 4 ). But here we have presented the density of states and band structures along the symmetry directions $\Gamma-\mathrm{X}-\mathrm{W}-\mathrm{L}-\mathrm{\Gamma}-\mathrm{K}$ corresponding to volume compressions $\mathrm{V} / \mathrm{Vo}=1.0$ and $\mathrm{V} / \mathrm{Vo}=0.3852$ for CdS only (Figs. 1 and 2).

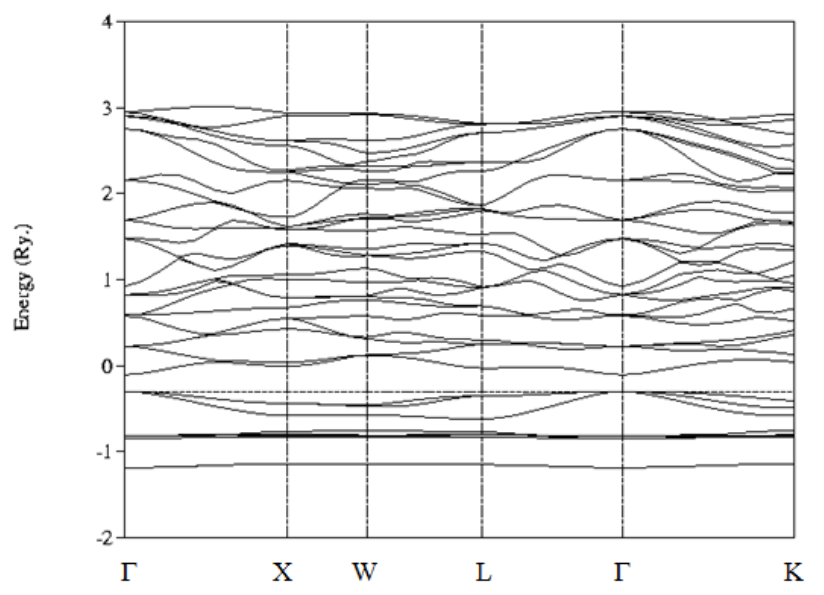

Figure 1. Normal pressure band structure of CdS ( $\mathrm{ZnS}$ structure) 


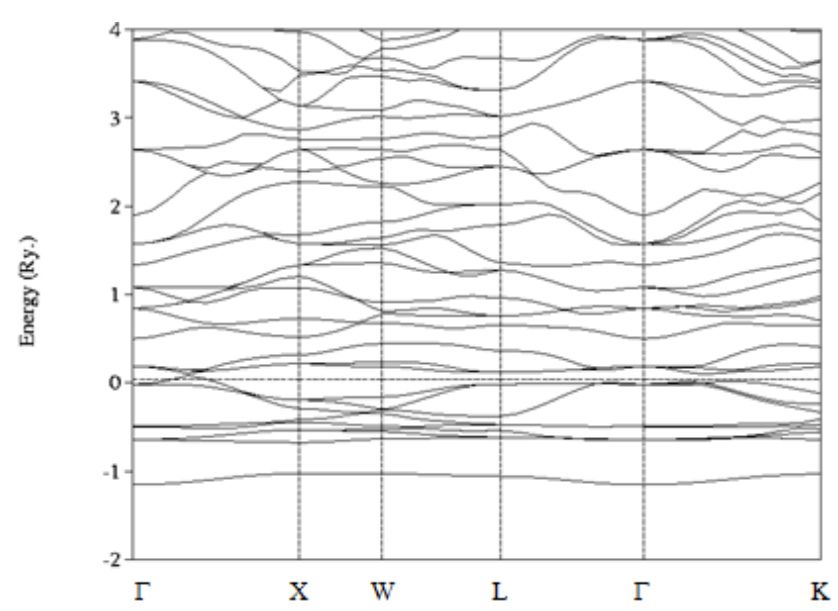

Figure 2. Metallization pressure band structure of $\mathrm{CdS}(\mathrm{NaCl}$ structure)

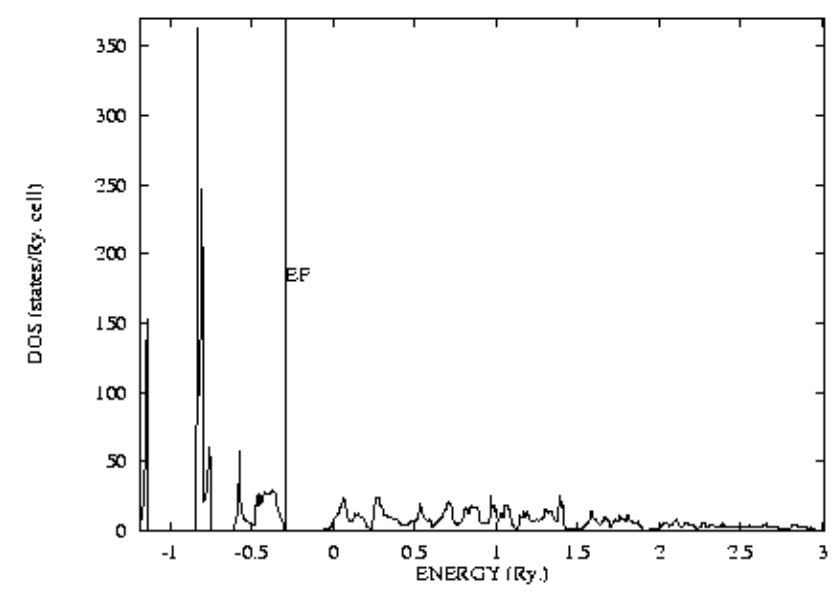

Figure 3. Normal pressure density of states of $\mathrm{CdS}$ ( $\mathrm{ZnS}$ structure)

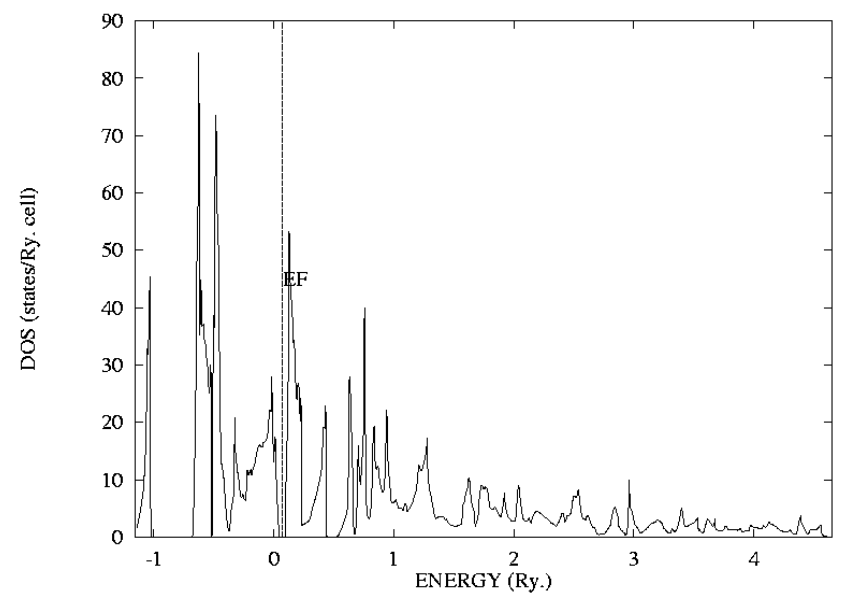

Figure 4. Metallization pressure Density of states of $\mathrm{CdS}(\mathrm{NaCl}$ structure)

In the bottom of the valence band, a single band is positioned due to $3 \mathrm{~s}^{2}$ electrons of S (Figs.1 and 2). Below the Fermi level, the three bands appearing due to the $5 \mathrm{~s}^{2}$ electrons of $\mathrm{Cd}$ and $3 \mathrm{p}^{4}$ electrons of $\mathrm{S}$ (Fig.1). The degenerate five bands are due to $4 \mathrm{~d}^{10}$ electrons of Cd. The $5 \mathrm{p}$, $5 \mathrm{~d}$ states of $\mathrm{Cd}$ and $3 \mathrm{~d}, 4 \mathrm{~s}$ states of $\mathrm{S}$ produces the empty conduction bands above the Fermi level (Fig.1). The direct band gap at normal pressure is found to be $2.42 \mathrm{eV}$ for $\mathrm{CdS}$ (at $\Gamma$ point). The increase in the width of the valence band and conduction band due to high pressure, leads to the narrowing of the band gap and finally metallization in $\mathrm{CdS}$ at $\mathrm{V} / \mathrm{Vo}=0.3852\left(\mathrm{P}_{\mathrm{M}}=6.9133 \mathrm{Mbar}\right)$ (Fig.2 and Table.1). Metallization occurs via direct closing of band gap in between $\Gamma$ and $X$ points (Fig.2). There is appreciable value for density of states at Fermi energy $N\left(E_{F}\right)$ at the metallization pressure. We have given the DOS histograms of CdS corresponding to normal pressure (Fig.3) and metallization pressure (Fig.4). At normal pressure (Fig. 3) the short spike near the origin is due to the levels arising from $3 \mathrm{~s}^{2}$ electrons of $\mathrm{S}$. The long spike near the Fermi energy is due to $3 p^{4}$ electrons of $\mathrm{S}$. The $5 \mathrm{p}$ and $5 \mathrm{~d}$ states of $\mathrm{Cd}$ and $\mathrm{S}$ produce the short peaks above the Fermi energy $E_{F}$. The width of the valence band and the empty conduction band get widened, as the pressure increases. These changes lead to the closing of the band gap under pressure (Fig.4).

Table 1. Metallization and phase transition pressures in CdS

\begin{tabular}{|c|c|c|c|c|}
\hline \multirow{2}{*}{$\begin{array}{c}\text { Cadmium } \\
\text { Compound }\end{array}$} & \multicolumn{2}{|c|}{ Metallization } & \multicolumn{2}{c|}{ Phase transition } \\
\cline { 2 - 5 } & $\begin{array}{c}\mathrm{P}_{\mathrm{M}} \\
\mathrm{Mbar}\end{array}$ & $(\mathrm{V} / \mathrm{Vo})_{\mathrm{M}}$ & $\begin{array}{c}\mathrm{P}_{\mathrm{T}} \\
\mathrm{Mbar}\end{array}$ & $(\mathrm{V} / \mathrm{Vo})_{\mathrm{T}}$ \\
\hline $\mathrm{CdS}$ & 6.9133 & 0.3852 & 0.0168 & 0.975 \\
\hline
\end{tabular}

When pressure increases ( reduced volume decreases)the value of the DOS at Fermi energy, $\mathrm{E}_{\mathrm{F}}\left(\mathrm{N}\left(\mathrm{E}_{\mathrm{F}}\right)\right.$ ) increases [2].The normal pressure DOS trend is changed under high pressure. The increase of pressure leads to the broadening of bands and decrease of density of states value in most of the energy regions. It also increases the width of the valence band and empty conduction bands [1,2]. When pressure increases, the value of $E_{F}$ increases whereas no density of states is available at the Fermi level up to metallization pressure. Further increase in pressure leads to enhanced density of states at the Fermi level which induces superconductivity [4].

\section{Results and Discussion}

\subsection{Ground State Properties}

From the total energies obtained from our calculation, the ground state properties and structural phase transition of $\mathrm{CdS}$ is studied. Pressure versus reduced volume curve for CdS is given in Fig.5. In this figure, when the volume decreases pressure increases. Pressure versus lattice constant curve for $\mathrm{CdS}$ is given in Fig.6. Here, when the pressure increases lattice constant decreases. Pressure versus band gap curve for $\mathrm{CdS}$ is given in Fig.7. In this figure, when the pressure increases band gap decreases and zero at high pressures. The total energy versus reduced volume curve is given in Fig.8. The total energy is calculated as a function of reduced volume (V/Vo) for both $\mathrm{ZnS}$ and $\mathrm{NaCl}$ phases of CdS. Here, Vo is the experimental equilibrium volume corresponding to the experimental equilibrium lattice constant. The calculated total energies were fitted to Murnaghan's equation of state [1] to obtain the pressure derivative of bulk modulus Bo' [2]. 
The calculated reduced volumes, lattice constant and band gap values of CdS for different pressure are given in Figs 5,6 and 7 respectively. From the total energy as a function of reduced volume curve (Fig.8), it is found that, in CdS, up to $\mathrm{V} / \mathrm{Vo}=0.975(\mathrm{PT}=0.0168 \mathrm{Mbar}), \mathrm{ZnS}$ structure has the lowest energy and on further reduction of volume $\mathrm{NaCl}$ structure becomes more stable in energy than the $\mathrm{ZnS}$ structure [18]. In Table 2, the equilibrium lattice constant $\left(\mathrm{a}_{\mathrm{o}}\right)$, band gap $\left(\mathrm{E}_{\mathrm{g}}\right)$, bulk modulus $\left(\mathrm{B}_{\mathrm{o}}\right)$ and its pressure derivative $\left(\mathrm{B}_{0}{ }^{1}\right)$ values are compared with experimental $[6,9]$ and previous theoretical works $[16,18]$. From Table 2, it is observed that one gets larger lattice constant corresponding to smaller bulk modulus [1]. Our estimated bulk modulus of $\mathrm{CdS}$ is less than that of $\mathrm{ZnS}$, as a result compressibility is higher for $\mathrm{CdS}$ than $\mathrm{ZnS}$. Thus, it is noted that the compressibility decreases as the size of the cation decreases. This is the general trend in alkali halides [19], which is confirmed now for CdS.

$$
\begin{aligned}
\mathrm{P} & =1.5 \mathrm{Bo}[(\mathrm{Vo} / \mathrm{V}) 7 / 3-(\mathrm{Vo} / \mathrm{V}) 5 / 3][1+ \\
& +0.75(\mathrm{Bo} 1-4)\{(\mathrm{Vo} / \mathrm{V}) 2 / 3-1\}]
\end{aligned}
$$

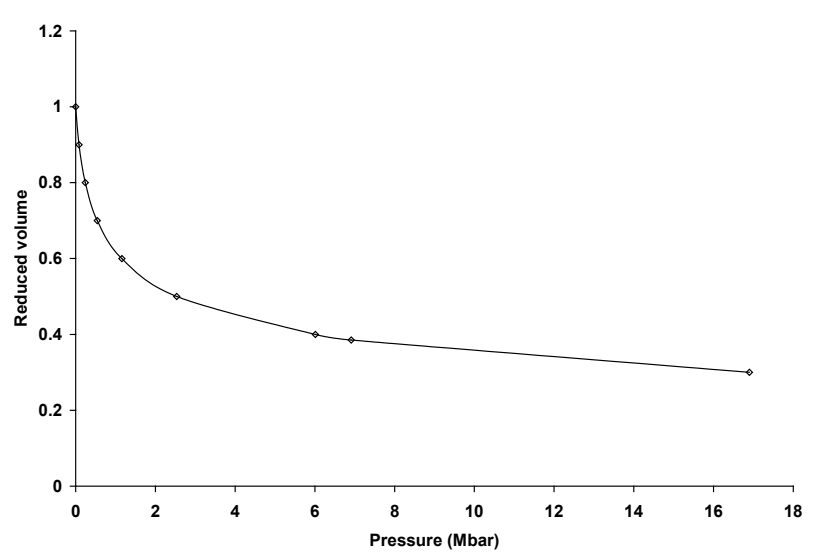

Figure 5. Pressure versus reduced volume curve for CdS

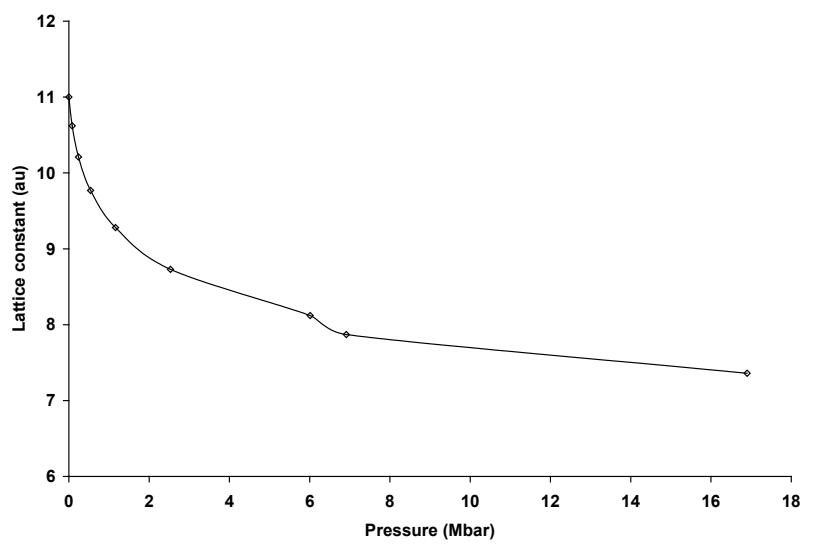

Figure 6. Pressure versus lattice constant curve for $\mathrm{CdS}$

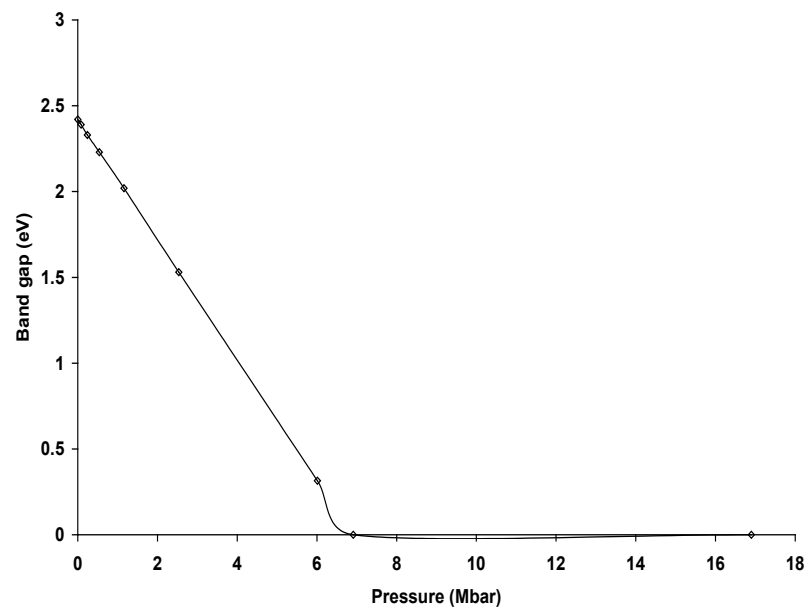

Figure 7. Pressure versus band gap curve for $\mathrm{CdS}$

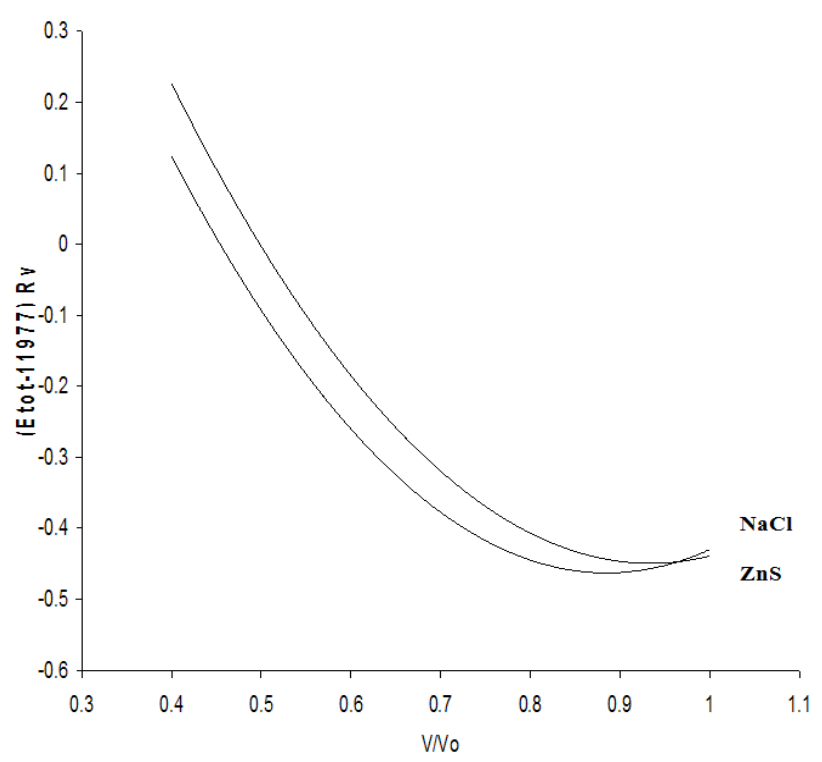

Figure 8. The total energy versus reduced volume curve for CdS

Table 2. Equilibrium lattice constant (ao), bulk modulus (Bo)and its pressure derivative $\left(\mathrm{Bo}^{1}\right)$ of $\mathrm{CdS}$ in $\mathrm{ZnS}$ structure

\begin{tabular}{|c|c|c|c|}
\hline \multirow{2}{*}{$\begin{array}{c}\text { Ground } \\
\text { State } \\
\text { Properties }\end{array}$} & \multicolumn{3}{|c|}{ CdS } \\
\cline { 2 - 4 } & $\begin{array}{c}\text { Present } \\
\text { work }\end{array}$ & $\begin{array}{c}\text { Experimental work } \\
{[6,9]}\end{array}$ & $\begin{array}{c}\text { Previous } \\
\text { theoretical works } \\
{[16,18]}\end{array}$ \\
\hline $\mathrm{a}_{\mathrm{o}}$ a.u. & 11.002 & 11.0 & 10.81 \\
\hline $\mathrm{B}_{\mathrm{o}} \mathrm{Mbar}$ & 0.72 & 0.73 & 0.69 \\
\hline $\mathrm{B}_{\mathrm{o}}{ }^{1}$ & 5.2 & 5.3 & 5.6 \\
\hline $\mathrm{E}_{\mathrm{g}} \mathrm{eV}$ & 2.42 & 2.45 & 2.402 \\
\hline
\end{tabular}




\subsection{Structural Phase Transition}

At ambient pressure $\mathrm{CdS}$ is in the $\mathrm{ZnS}$ structure. The phase stability of the $\mathrm{B} 3(\mathrm{ZnS})$ and $\mathrm{B} 1(\mathrm{NaCl})$ structures of $\mathrm{CdS}$ is analyzed using the enthalpy calculation [20,21]. The enthalpy $\mathrm{H}(\mathrm{P})$ is defined by

$$
\mathrm{H}(\mathrm{P})=\text { Etot }(\mathrm{P})+\mathrm{PV}(\mathrm{P})
$$

and the transition pressure corresponding to the phase transitionfromB3 to $\mathrm{B} 1$ is obtained from the relation

$$
\text { HB3 }(\mathrm{P})=\mathrm{HB} 1(\mathrm{P})
$$

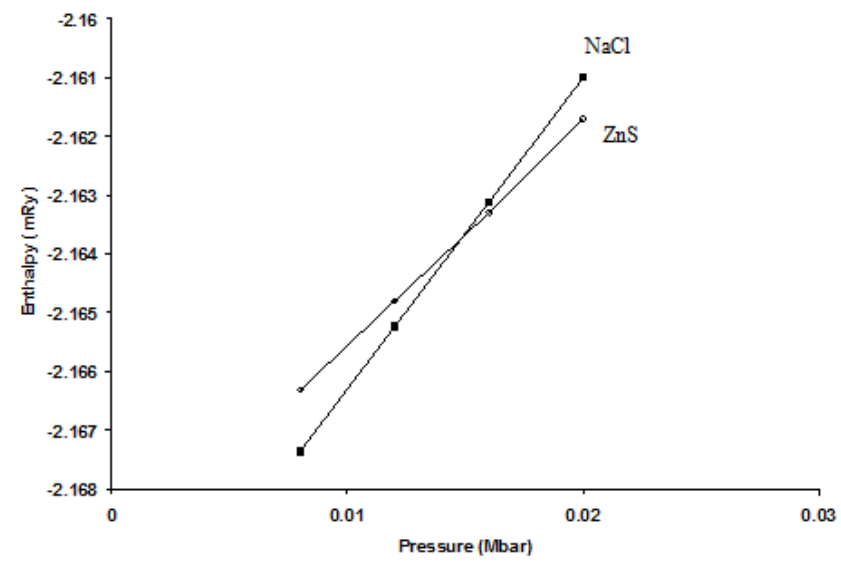

Figure 9. The pressure versus enthalpy curve for $\mathrm{CdS}$

where HB3 and HB1 are the enthalpies of the B3 and B1 phases respectively. The pressure versus enthalpy curve for $\mathrm{CdS}$ is given in Fig.9.

From the enthalpy calculation, the phase transition pressure $\left(\mathrm{P}_{\mathrm{T}}=0.0168 \mathrm{Mbar}\right)$ and the corresponding reduced volume $\left((\mathrm{V} / \mathrm{Vo})_{\mathrm{T}}=0.975\right)$ are estimated for $\mathrm{CdS}($ Table.1). Our calculated phase transition pressure of CdS supports the experimental value of 0.02 Mbar [6]). The mechanism for the phase transition is a geometric effect involving a change in the coordination number from 4 in the $\mathrm{ZnS}$ phase to 6 in the $\mathrm{NaCl}$ phase $[22,23]$.

\subsection{Metallization}

At normal pressure, $\mathrm{CdS}$ is a direct band gap semiconductor [24,25]. The band gap decreases with the increase of the pressure and at a particular pressure, there is a closing of the band gap. The band structure corresponding to metallization of $\mathrm{CdS}$ is shown in Fig. 3. In $\mathrm{CdS}$, the metallization occurs through direct closing of the band gap inbetween $\Gamma$ and $X$ points (Fig. 2). In $\mathrm{CdS}$, the direct closure of the band gap inbetween $\Gamma$ and $X$ point takes place at the reduced volume $\mathrm{V} / \mathrm{Vo}=0.3852(\mathrm{NaCl}$ structure $)$, the corresponding metallization pressure is 6.9133Mbar (Fig.2). For CdS, no experimental metallization value available for comparison. But our calculation is in agreement with the simple linear extrapolation data of Cervantes etal [7]. They estimated the metallization pressure of $\mathrm{NaCl}$-structured $\mathrm{CdS}$ was above $250 \mathrm{GPa}$ or $2.5 \mathrm{Mbar}$. Because phase transition occurs below this pressure and $\mathrm{NaCl}$-structured phase will undergo metallization [7]. Similar to group III-V compounds [22], prior to metallization, group II-VI compounds undergo structural transition from $\mathrm{ZnS}$ to $\mathrm{NaCl}$ structure $[1,4]$. The values for density of states at Fermi energy $N\left(E_{F}\right)$ are very small, at the metallization pressures, which indicate that metallization has just set in CdS (Fig. 4). Thereafter $\mathrm{N}\left(\mathrm{E}_{\mathrm{F}}\right)$ increases slowly with pressure and becomes fairly large at a particular value of V/Vo [20]. The values of $E_{F}$ and $N\left(E_{F}\right)$ corresponding to different $\mathrm{V} / \mathrm{Vo}$ are used in studying the pressure variation of superconducting transition temperature [26-29].

\section{Conclusions}

In summary, we have investigated the pressure dependent band structure, density of states, structural phase transition and metallization of cubic zinc blende type $\mathrm{CdS}$ using FP-LMTO method. Cadmium sulphide becomes metal under high pressure, but before that they undergo structural phase transition from $\mathrm{ZnS}$ to $\mathrm{NaCl}$ structure. The ground state properties and band gap values are compared with the experimental and previous theoretical results. When the pressure is increased there is enhanced overlapping between the wave functions of the neighbouring atoms. As a result the widths of the valence and empty conduction bands increase. The broadening of both the valence and conduction bands with pressure leads to the band gap closure and the formation of metallic states [26]. These changes lead to the narrowing and closing of band gaps in $\mathrm{CdS}$ (metallization). Our calculated phase transition pressure of $\mathrm{CdS}$ is $(0.0168$ Mbar) agreement with the experimental value of $0.02 \mathrm{Mbar}$. The mechanism for the phase transition is a geometric effect involving a change in the coordination number from 4 in the $\mathrm{ZnS}$ phase to 6 in the $\mathrm{NaCl}$ phase [27]. In $\mathrm{CdS}$, the direct closure of the band gap in-between $\Gamma$ and $\mathrm{X}$ point takes place at the reduced volume $\mathrm{V} / \mathrm{Vo}=0.3852(\mathrm{NaCl}$ structure $)$, the corresponding metallization pressure is 6.9133Mbar. For $\mathrm{CdS}$, no experimental metallization value available for comparison. It is also confirmed that the metallization and structural phase transition do not occur simultaneously in CdS.

\section{REFERENCES}

[1] J. Jesse Pius and C. Nirmala Louis, Chemical and Materials Engineering 4(3): 46-56, (2016).

[2] V. Benaline Sheeba and C. Nirmala Louis, International Journal of Scientific Research and Innovations, 1, 1-5 (2016).

[3] M. I. Eremets, K. Shimizu, and K. Amaya, Science 281, 1333 (1998).

[4] Y. Wang, J. Zhang, C. Wang, C. Li , C. Gao, Chalcogenide Letters 12(7),357 (2015). 
[5] J.J. Tan, Y. Li and G.F. Ji, Acta Physica Polonica A, 120 (3), $501(2011)$

[6] Vladimir V. Shchennikov, Sergey V. Ovsyannikov, Physica status solidi (b), 244 (1), 437(2007).

[7] P. Cervantes, Q. Williams, M. Cote, O. Zakharovand M.L. Cohen, Phys. Rev. B, 54 (24), 17585 (1996).

[8] Mujica et al.: High-pressure phases of group-IV, III-V, and II-VI compounds Rev. Mod. Phys., Vol. 75, No. 3 (2003)

[9] Edwards, A. L., and H. G. Drickamer, Phys. Rev. 122, 1149 (1961).

[10] O. K. Andersen, O. Jepsen and M. Sob, "Elctronic band structure and its applications", edited by M. Yussouff (Springer, Heidelberg, 1987)

[11] Mariano, A. N., and E. P. Warekois, Science 142, 672 (1963).

[12] Rooymans, C. J. M., Phys. Lett. 4, 186(1963).

[13] Suzuki, T., T. Yagi, S. Akimoto, T. Kawamura, S. Toyoda, and S. Endo, J. Appl. Phys. 54, 748 (1983).

[14] Nelmes, R. J., and M. I. McMahon, Semicond. Semimetals, 54, 145(1998).

[15] Co te, M., O. Zakharov, A. Rubio, and M. L. Cohen, Phys. Rev. B 55, 13 025(1997).

[16] Dreysse, H., Ed., Electronic Structure and Physical Properties of Solids: The Uses of the FP-LMTO Method (Springer, Berlin) (2000).
[17] Holzapfel, W. B., and N. S. Isaacs, Eds, High-Pressure Techniques in Chemistry and Physics (Oxford University Press,) (1997).

[18] O. M. Madelung, Semiconductors: Data Handbook, 3rd ed. Springer, Berlin, (2004).

[19] C. Nirmala Louis and K. Iyakutti, Phys. Stat. Sol (b) 236, 614 (2003); Phys. Stat. Sol (b) 233, 339 (2002).

[20] S.Q. Wang and H.Q. Ye, Phys. Rev. B 66, 235111 (2002).

[21] C. Nirmala Louis, A. AmalRaj and K. Iyakutti, Journal of theoretical and computational Chemistry, vol.11, pg. 19-33 (2012).

[22] V. Benaline Sheeba and C. Nirmala Louis, Chemical and Materials Engineering 1(4): 132-140, 2013.

[23] A. Vladimir and R. Serget, Physica status solidi (b), Vol.244, 243-248 (2007).

[24] S.Q Wang and H.Q Ye, Journal of physics: condensed matter, Vol.14, 9579-9587 (2002).

[25] D. Fritsch, H. Schmidt and M. Grundmann Phys. Rev. B, 67, 235205 (2003).

[26] S.S. Saxena and P. Monthoux, Nature 427, 799 (2004).

[27] N. E. Christensen and I. Gorczyca, Phys. Rev. B 47, 4307 (1993).

[28] I. Vurgaftman, J.R. Meyer and L.R. Ram Mohan, J. Appl. phys. 89, 5815(2001).

[29] S. Froyen and M.L. Cohen, Phys. Rev. B, 28, 3258 (1983). 